\title{
Variações temporais e espaciais na composição e estrutura da comunidade de peixes jovens da Baía de Sepetiba, Rio de Janeiro
}

\author{
André Luiz Machado Pessanha ${ }^{1}$ \\ Francisco Gerson Araújo 1 \\ Márcia Cristina Costa de Azevedo 1 \\ Iracema David Gomes ${ }^{1}$
}

\begin{abstract}
Spatial and seasonal variations in community fish structure of juvenile fish in Sepetiba Bay, Rio de Janeiro. A year-long beach seine survey at the continental margin of Sepetiba Bay, Rio de Janeiro State, Brazil, catching mainly young-of-the-year and juvenile fishes was carried out from July 1983 to June 1984, in order to describe the community structure and their spatial and seasonal variations. Fifty-five monthly samplied carried out in five sites in the marginal surf zone waters yielded 11,463 fishes in 82 species, 59 genera and 31 families. Gerreidae, Engraulididae, Atherinidae and Ariidae families amounted $87.01 \%$ of the total catch in number, and $81 \%$ in weight. Gerres aprion Cuvier, 1829, Anchoa januaria (Steindachner, 1879), Anchoviella lepidentostole (Fowler, 1911), Atherinella brasiliensis (Quoy \& Gaimard, 1824) and Netuma barba (Lacépède, 1803) were the most numerous species, each one contributing more the $4 \%$ of total catch. Overall, both spatial and seasonal changes in fish were not evident although most individuals were more abundant in the outer Bay. The high number of fish species in this area, suggest the important role played by the Sepetiba Bay in the fish community to where many species probably migrate from the nearby sea and stay in the Bay during part of their life cycle. Decreasing diversity and high dominance of a few number of species seem to indicated a trend of environmental deterioration in the Sepetiba Bay over the last decade, as this date are compared whith similar sampling program carried out in 1993/94. It same to indicated that changes in fish populations which use this area as rearing grounds, occurred during their early life history.

KEY WORDS. Sepetiba Bay, ichthyofauna, fish, bioecology
\end{abstract}

A região litorânea na qual se encontram enseadas, baías, lagunas costeiras e estuários é reconhecidamente uma área de proteção, alimentação e reprodução para um número considerável de espécies de peixes, funcionando como habitat temporário durante fases do ciclo de vida ou ainda como habitat permanente para outras espécies (HORN 1980; LIVINGSTON 1982; BENNET 1989; DAY et al. 1989).

A Baía de Sepetiba, um dos importantes ecossistemas aquáticos do estado do Rio de Janeiro, abriga um grande número de peixes em seus primeiros anos de vida, ou espécies de pequeno porte que ocorrem na zona de arrebentação (ARAÚJO et al. 1997). Nos últimos anos uma considerável pressão urbana-industrial vem 
contribuindo para mudanças ambientais de sua margem continental que influenciam diretamente para alteração deste ecossistema costeiro. A biota aquática, e em particular a comunidade de peixes como organismos associados diretamente às condições ambientais, podem estar sendo alteradas em função de tais mudanças.

Poucos são os trabalhos que enfocam a sua margem continental, destacando estudos feitos por Oshiro \& ARAújo (1987), SERGIPENSE \& SAZIMA (1995), SANTOS et al. (1997) e ARAÚJO et al. (1997).

No presente são analisadas a estrutura da comunidade da margem continental da Baía de Sepetiba entre julho de 1983 e junho de 1984, ressaltando aspectos dos padrões de distribuição espacial e temporal das populações de peixes. Comparações com similar programa de amostragem realizado cobrindo um mesmo ciclo anual (julho 1993 a junho de 1994) nas mesmas estações de coleta (ARAÚJo et al. 1997) visando apresentar uma tendência da estrutura e comunidade de peixes foram realizadas.

\section{MATERIAL E MÉTODOS}

Um total de 55 amostragens de arrastos de praia foram realizados na margem continental da Baía de Sepetiba ( $22^{\circ} 54^{\prime}-23^{\circ} 04^{\prime} \mathrm{S}$ e $\left.43^{\circ} 34^{\prime}-44^{\circ} 10^{\prime} \mathrm{W}\right)$ entre julho de 1983 e junho de 1984. Excepcionalmente, não foram feitas as amostragens do mês de janeiro de 1984. Os arrastos foram efetuados paralelos a linha da costa, utilizando uma rede do tipo beach seine, operada manualmente e medindo $10 \mathrm{~m}$ de comprimento, $2,5 \mathrm{~m}$ de altura e malha de $7 \mathrm{~mm}$ de distâncias entrenós, com uma extensão de aproximadamente $50 \mathrm{~m}$, percorrendo uma área de $500 \mathrm{~m}^{2}$, em cinco locais de coleta definidas de acordo com sua localização, sendo duas na zona externa [Praia de Muriqui (MU); Praia de Itacuruçá (IT)) e três na zona interna (Coroa Grande (CG); Praia de Sepetiba (PS); Pedra de Guaratiba (PG)].

Os peixes coletados foram fixados em formol $10 \%$ e conduzidos a laboratório, onde foram realizadas a identificação, contagem e pesagem em gramas. Os peixes foram identificados segundo CARVALHO et al. (1968), FISCHER (1978), Figueiredo \& MENEZES $(1978,1980)$, MENEZES \& FigueIREDo $(1980,1985)$ e ANDREATA $(1988,1989)$. A distribuição e abundância relativa das espécies foram calculadas com base no somatório do número e do peso dos indivíduos capturados, dividido pelo total de arrastos.

Para avaliação das variações espaciais e temporais foi feita Análise de Variância Bifatorial aplicada sobre o número e peso de peixes, número de espécies e abundância das espécies de maior ocorrência visando comparar suas médias. A normalidade e a homocedasticidade das variáveis, foram determinadas através do teste de Bartlet (SOKAL \& ROHLF 1981), e como a maioria dos dados não atendeu a estes requisitos, foi utilizada a transformação logarítmica $\log (x+1)$ onde $\log$ é o logaritmo na base 10, e x é o valor não transformado. Tais transformações foram feitas previamente à análise de variância, a qual foi seguida do teste de diferenças de médias de Student-Newman-Keuls (SNK) ao nível de confiança de 95\% ( $\mathrm{p}<$ 0,05 ), para determinação de quais médias são significantemente diferentes, toda vez que a hipótese zero foi rejeitada. 
As variações espaciais foram feitas considerando as estações de coleta. $\mathrm{O}$ critério de sazonalidade (período do ano) foi feito considerando os meses de amostragem, no seguinte agrupamento: verão (dezembro, janeiro e fevereiro), outono (março, abril e maio), inverno (junho, julho e agosto) e primavera (setembro, outubro e novembro).

\section{RESULTADOS}

\section{Composição e Estrutura}

Foram capturadas nas 55 amostragens de arrasto de praia, 82 espécies de peixes compreendidos em 59 gêneros e 31 famílias, totalizando 11.463 indivíduos e $15.821 \mathrm{~g}$ de peixes no período estudado (Tab. I). As espécies foram em sua grande maioria juvenis em seu primeiro ano de vida ou espécies de pequeno tamanho que habitam a margem continental da Baía.

Tabela I. Número total de peixes capturados na Baía de Sepetiba, Rio de Janeiro, julho 1983 a junho 1984, sumarizados por famílias. (FO) Freqüências de ocorrência (\%).

\begin{tabular}{|c|c|c|c|c|c|}
\hline Familias & Espécies & $\begin{array}{l}\mathrm{N}^{\circ} \text { de ordem de } \\
\text { abundância }\end{array}$ & $\begin{array}{c}\mathrm{N}^{\circ} \text { de peixes } \\
\text { coletados }\end{array}$ & $\begin{array}{l}\text { Porcentagem } \\
\text { do total }\end{array}$ & $\begin{array}{l}\text { FO } \\
\text { (\%) }\end{array}$ \\
\hline Muraneidae & Gymnothorax ocellatus Agasiz, 1831 & 62 & 2 & $<0,1$ & 2,5 \\
\hline \multirow[t]{2}{*}{ Clupeidae } & Harengula clupeola (Cuvier, 1829) & 10 & 158 & 1,4 & 7,5 \\
\hline & Platanicthys platana(Regan, 1917) & 31 & 14 & 0,1 & 2,5 \\
\hline \multirow[t]{6}{*}{ Engraulididae } & Anchoa januaria (Steindachner, 1879) & 2 & 2337 & 20,4 & 30,0 \\
\hline & Anchoa tricolor (Agassiz, 1829) & 9 & 171 & 1,5 & 17,5 \\
\hline & Anchoa lyolepis (Everman \& Marsh, 1902) & 65 & 2 & $<0,1$ & 1,3 \\
\hline & Anchoviella lepidentostole (Fowler, 1911) & 3 & 1542 & 13,5 & 11,2 \\
\hline & Cetengraulis edentulus (Cuvier, 1828) & 27 & 20 & $<0,1$ & 2,5 \\
\hline & Lycengraulis grossidens (Agassiz, 1829) & 24 & 29 & 0,2 & 6,2 \\
\hline \multirow[t]{3}{*}{ Ariidae } & Netuma barba (Lacépède, 1803) & 5 & 532 & 4,6 & 15,0 \\
\hline & Genidens genidens (Valenciennes, 1839) & 11 & 134 & 1,2 & 8,8 \\
\hline & Cathorops spixii (Agassiz, 1829) & 14 & 116 & 1,0 & 11,3 \\
\hline Synodontidae & Synodus foetens (Linnaeus, 1766) & 46 & 4 & $<0,1$ & 1,3 \\
\hline \multirow[t]{2}{*}{ Exocoetidae } & Hemiramphus brasiliensis (Linnaeus, 1758) & 71 & 1 & $<0,1$ & 2,5 \\
\hline & Hyporhamphus unifasciatus (Ranzani, 1842) & 59 & 2 & $<0,1$ & 2,5 \\
\hline Belonidae & Strongylura timicu (Walbaum, 1792) & 36 & 7 & $<0,1$ & 5,0 \\
\hline Poecilidae & Poecilia vivipara (Schneider, 1801) & 23 & 29 & 0,3 & 6,3 \\
\hline Gobiesocidae & Gobiesox strumosus Cope, 1870 & 72 & 1 & $<0,1$ & 1,3 \\
\hline Atherinidae & Atherinella brasiliensis (Quoy \& Gaimard, 1824) & 4 & 1144 & 10,0 & 52,5 \\
\hline \multirow[t]{2}{*}{ Syngathidae } & Syngnathus folletti Herald, 1942 & 37 & 7 & $<0,1$ & 2,5 \\
\hline & Hippocampus reidi Ginsburg, 1933 & 60 & 2 & $<0,1$ & 2,5 \\
\hline Triglidae & Prionotus punctatus (Bloch, 1797) & 35 & 8 & $<0,1$ & 3,8 \\
\hline Centropomidae & Centropomus undecimalis (Bloch, 1792) & 76 & 1 & $<0,1$ & 1,3 \\
\hline \multirow[t]{2}{*}{ Serranidae } & Diplectrum radiale (Quoy \& Gaimard, 1824) & 75 & 1 & $<0,1$ & 1,3 \\
\hline & Serranus phoebe Poey, 1851 & 66 & 1 & $<0,1$ & 1,3 \\
\hline Pomatomidae & Pomatomus saltator (Linnaeus, 1766) & 58 & 2 & $<0,1$ & 1,3 \\
\hline \multirow[t]{7}{*}{ Carangidae } & Trachinotus falcatus (Linnaeus, 1758) & 25 & 26 & 0,2 & 13,8 \\
\hline & Trachinotus carolinus (Linnaeus, 1766) & 29 & 16 & 0,1 & 10,0 \\
\hline & Trachinotus goodei Jordan \& Evermann, 1896 & 56 & 2 & $<0,1$ & 1,3 \\
\hline & Oligoplistes palometa (Cuvier, 1833) & 31 & 14 & 0,1 & 2,5 \\
\hline & Oligoplistes saurus (Bloch \& Schneider, 1801) & 32 & 12 & 0,1 & 5,0 \\
\hline & Selene vomer (Linnaeus, 1758) & 57 & 2 & $<0,1$ & 1,3 \\
\hline & Caranx latus Agassiz, 1831 & 54 & 3 & $<0,1$ & 2,5 \\
\hline \multirow[t]{7}{*}{ Gerreidae } & Diapterus rhombeus (Cuvier, 1829) & 21 & 36 & 0,3 & 6,2 \\
\hline & Diapterus auratus Ranzani, 1840 & 42 & 6 & $<0,1$ & 2,5 \\
\hline & Diapterus lineatus (Humboldt \& Valenciennes, 1811) & 7 & 291 & 2,5 & 1,3 \\
\hline & Gerres aprion Cuvier, 1829 & 1 & 3524 & 30,7 & 41,3 \\
\hline & Gerres gula Quoy \& Gaimard, 1824 & 41 & 6 & $<0,1$ & 3,8 \\
\hline & Gerres lefroyi (Goode, 1874) & 15 & 85 & 0,7 & 11,3 \\
\hline & Gerres melanopterus Bleeker, 1859 & 38 & 7 & $<0,1$ & 2,5 \\
\hline
\end{tabular}


Tabela I. Continuação.

\begin{tabular}{|c|c|c|c|c|c|}
\hline Familias & Espécies & $\begin{array}{l}\mathrm{N}^{\circ} \text { de ordem de } \\
\text { abundância }\end{array}$ & $\begin{array}{c}\mathrm{N}^{\circ} \text { de peixes } \\
\text { coletados }\end{array}$ & $\begin{array}{l}\text { Porcentagem } \\
\text { do total }\end{array}$ & $\begin{array}{l}\text { FO } \\
\text { (\%) }\end{array}$ \\
\hline \multirow[t]{5}{*}{ Haemulidae } & Boridia grossidens (Cuvier, 1830) & 17 & 46 & 0,4 & 2,5 \\
\hline & Conodon nobilis (Linnaeus, 1758) & 78 & 1 & $<0,1$ & 1,3 \\
\hline & Haemulum steidachneri (Jordan \& Gilbert, 1882) & 44 & 5 & $<0,1$ & 1,3 \\
\hline & Orthopristis ruber (Cuvier, 1830) & 69 & 1 & $<0,1$ & 1,3 \\
\hline & Pomadasys ramosus (Poey, 1860) & 40 & 6 & $<0,1$ & 1,3 \\
\hline Sparidae & Diplodus argenteus (Valenciennes, 1830) & 13 & 119 & 1,0 & 2,5 \\
\hline \multirow[t]{10}{*}{ Sciaenidae } & Cynoscion leiarchus (Cuvier, 1830) & 53 & 3 & $<0,1$ & 2,5 \\
\hline & Menticirrhus americanus (Linnaeus, 1758) & 20 & 37 & 0,3 & 13,8 \\
\hline & Menticirrhus littoralis (Holbrook, 1860) & 18 & 45 & 0,4 & 8,7 \\
\hline & Micropogonias furmieri (Desmarest, 1823) & 6 & 292 & 2,6 & 18,8 \\
\hline & Ophioscion punctatissimus Meek \& Hildebrand, 1925 & 19 & 41 & 0,3 & 8,7 \\
\hline & Umbrina coroides (Cuvier, 1830) & 8 & 237 & 2,1 & 7,5 \\
\hline & Stellifer rastrifer (Jordan, 1889) & 43 & 12 & $<0,1$ & 1,0 \\
\hline & Stellifer stellifer (Bloch, 1790) & 48 & 3 & $<0,1$ & 2,5 \\
\hline & Bairdiella ronchus (Cuvier, 1830) & 34 & 9 & $<0,1$ & 1,3 \\
\hline & Odontoscion dentex (Cuvier, 1830) & 70 & 1 & $<0,1$ & 1,3 \\
\hline Ephippididae & Chaetodipterus faber (Broussonet, 1782) & 79 & 1 & $<0,1$ & 1,3 \\
\hline \multirow[t]{5}{*}{ Mugilidae } & Mugil liza Valenciennes, 1836 & 51 & 3 & $<0,1$ & 2,5 \\
\hline & Mugil curema Valenciennes, 1836 & 16 & 65 & 0,6 & 11,2 \\
\hline & Mugil gaimardianus Desmarest, 1831 & 22 & 30 & 0,3 & 7,5 \\
\hline & Mugil curvidens Valenciennes, 1836 & 43 & 5 & $<0,1$ & 2,5 \\
\hline & Mugil trichodon Valenciennes, 1836 & 49 & 3 & $<0,1$ & 1,3 \\
\hline Polynemidae & Polydactylus virginicus (Linnaeus, 1758) & 68 & 1 & $<0,1$ & 1,3 \\
\hline Dactyloscopidae & Dactyloscopus crossotus Starks, 1913 & 63 & 2 & $<0,1$ & 2,5 \\
\hline \multirow[t]{7}{*}{ Gobiidae } & Gobionellus oceanicus (Pallas, 1770) & 45 & 5 & $<0,1$ & 2,5 \\
\hline & Gobionellus stigmaticus (Poey, 1861) & 61 & 2 & $<0,1$ & 1,3 \\
\hline & Gobionellus stomatus Starks, 1913 & 73 & 1 & $<0,1$ & 1,3 \\
\hline & Gobionelus boleosoma (Jordan \& Gilbert, 1882) & 28 & 17 & 0,2 & 5,0 \\
\hline & Bathygobius soparator (Valenciennes, 1837) & 55 & 3 & $<0,1$ & 2,5 \\
\hline & Evorthodus lyricus (Girard, 1858) & 52 & 3 & $<0,1$ & 2,5 \\
\hline & Microgobius meeki Evermann \& Marsh, 1900 & 50 & 3 & $<0,1$ & 3,8 \\
\hline \multirow[t]{4}{*}{ Bothidae } & Etropus longimanus Norman, 1933 & 74 & 1 & $<0,1$ & 1,3 \\
\hline & Citharichthys spilopterus Günther, 1862 & 30 & 15 & 0,1 & 7,5 \\
\hline & Citharichthys arenaceus Evermann \& Marsh, 1902 & 47 & 4 & $<0,1$ & 2,5 \\
\hline & Citharichthys cornutus (Günther, 1880) & 64 & 2 & $<0,1$ & 2,5 \\
\hline Soleidae & Achirus declivis Chabanaud, 1940 & 26 & 21 & 0,2 & 11,3 \\
\hline \multirow[t]{2}{*}{ Cynoglossidae } & Symphurus diomedianus (Goode \& Bean, 1885) & 67 & 1 & $<0,1$ & 1.3 \\
\hline & Symphurus plagusia (Linnaeus, 1766) & 39 & 6 & $<0,1$ & 2,5 \\
\hline Balistidae & Balistes capriscus (Gmelin, 1788) & 80 & 1 & $<0,1$ & 1,3 \\
\hline \multirow[t]{2}{*}{ Tetraodontidae } & Sphoeroides testudineus (Linnaeus, 1758) & 12 & 129 & 1,2 & 18,8 \\
\hline & Sphoeroides spengleri (Bloch, 1785) & 68 & 3 & $<0,1$ & 2,1 \\
\hline Diodontidae & Chilomycterus spinosus (Linnaeus, 1758) & 77 & 1 & $<0,1$ & 1,0 \\
\hline
\end{tabular}

Do total de 31 famílias capturadas, 4 (Gerreidae, Engraulididae, Atherinidae e Ariidae) representaram $87,01 \%$ em número e $81,02 \%$ em peso do total de peixes capturados (Tab. II), sendo que Sciaenidae, Carangidae e Gerreidae foram as famílias com maior número de espécies, com 10, 7 e 7 espécies, respectivamente.

Tabela II. Percentual em número e peso das famílias de peixes mais abundantes na margem continental da Baia de Sepetiba, Rio de Janeiro, entre julho de 1983 e junho de 1984.

\begin{tabular}{lclc}
\hline Familias & Número $(\%)$ & Familias & Peso $(\%)$ \\
\hline Engraulididae & 35,77 & Gerreidae & 25,80 \\
Gerreidae & 34,44 & Aridae & 22,15 \\
Atherinidae & 9,98 & Engraulididae & 20,45 \\
Ariidae & 6,82 & Atherinidae & 12,62 \\
Outras & 12,99 & Outras & 18,98 \\
\hline
\end{tabular}


Cinco espécies, sendo duas da família Engraulididae (Anchoa januaria e Anchoviella lepidentostole), uma de Gerreidae (Gerres aprion), uma de Atherinidae (Atherinella brasiliensis) e uma de Ariidae (Netuma barba) representaram 79,2\% do número total de peixes, enquanto que duas espécies de Ariidae (Cathorops spixii e Netuma barba), uma de Gerreidae (Gerres aprion), uma de Engraulididae (Anchoa januaria), uma de Atherinidae (Atherinella brasiliensis) representaram $63,79 \%$ do peso total capturado (Tab. III). Das 80 espécies capturadas, 14 representaram $93,58 \%$ da captura total em número.

As cinco espécies mais abundantes foram: G. aprion, A. januaria, A. lepidentostole, A. brasiliensis e $N$. barba, sendo que cada uma delas contribuiu acima de $4 \%$ do número total de peixes capturados. Quinze espécies ocorreram apenas uma vez nas capturas.

Tabela III. Percentual em número e peso das das espécies mais abundantes na margem continental da Baía de Sepetiba, Rio de Janeiro, entre julho de 1983 e junho de 1984.

\begin{tabular}{lclc}
\hline \multicolumn{1}{c}{ Espécies } & Número $(\%)$ & \multicolumn{1}{c}{ Espécies } & Peso (\%) \\
\hline Gerres aprion & 30,74 & Gerres aprion & 18,54 \\
Anchoa januaria & 20,39 & Anchoa januaria & 12,92 \\
Anchoviella lepidentostole & 13,45 & Netuma barba & 12,74 \\
Atherinella brasiliensis & 9,98 & Atherinella brasiliensis & 12,62 \\
Netuma barba & 4,64 & Cathorops spixii & 6,97 \\
Outras & 20,80 & Outras & 36,21 \\
\hline
\end{tabular}

\section{Variações Temporais e Espaciais}

Significantes variações no número de peixes e no número de espécies por arrasto foram encontrados entre os meses amostrados. $\mathrm{O}$ número de peixes foi significativamente maior $(\mathrm{F}=2,1 ; \mathrm{p}<0,05)$ nos meses de maio-junho e dezembro-fevereiro (345-473 indivíduos/arrasto) e menor nos meses de agosto-setembro-outubro e abril (14-59 indivíduos/arrasto). O número de espécies foi significativamente maior $(\mathrm{F}=6,3 \mathrm{e} \mathrm{p}<0,01)$ nos meses de fevereiro-julho (9,6-9,8 espécies/arrasto) do que nos meses de agosto-setembro (2,6-5,0 espécies/arrasto) (Tab. IV; Fig. 1). Os maiores registros de espécies foram verificados no verão e no outono para as estações localizadas na zona interna da Baía (estação $4=$ PG e estação $5=$ PS) e o número total de peixes foi maior na estação da zona interna $(5=\mathrm{PG})$, durante o verão.

Tabela IV. Valores de F da Análise de Variância Bifatorial sobre a abundância no número, peso de peixes e número de espécies mais abundantes.

\begin{tabular}{lccc}
\hline \multicolumn{1}{c}{ Variáveis } & Estação do ano $(\mathrm{GL}=3)$ & Estação de coleta $(\mathrm{GL}=4)$ & Interaçăo $(\mathrm{GL}=12)$ \\
\hline Individuos & $3,5^{*}$ & 0,5 & 0,6 \\
Espécies & $3,6^{*}$ & 0,5 & 0,3 \\
Peso & 2,3 & 0,9 & 0,9 \\
Gerres aprion & $10,3^{* *}$ & $12,1^{* *}$ & $2,4^{*}$ \\
Anchoa januaria & $2,8^{*}$ & $5,3^{* *}$ & 0,7 \\
Anchoviella lepidentostole & 1,4 & $0,8^{*}$ & 0,9 \\
Atherinella brasiliensis & $3,2^{*}$ & $2,1^{*}$ & 0,6 \\
Netuma barba & 1,8 & 2,9 & 0,5 \\
\hline
\end{tabular}

(*) $\mathrm{P}<0,05 ;\left(^{\star \star}\right) \mathrm{P}<0,01 ;(\mathrm{GL})$ graus de liberdade; Interação = EA versus $\mathrm{EC}$. 


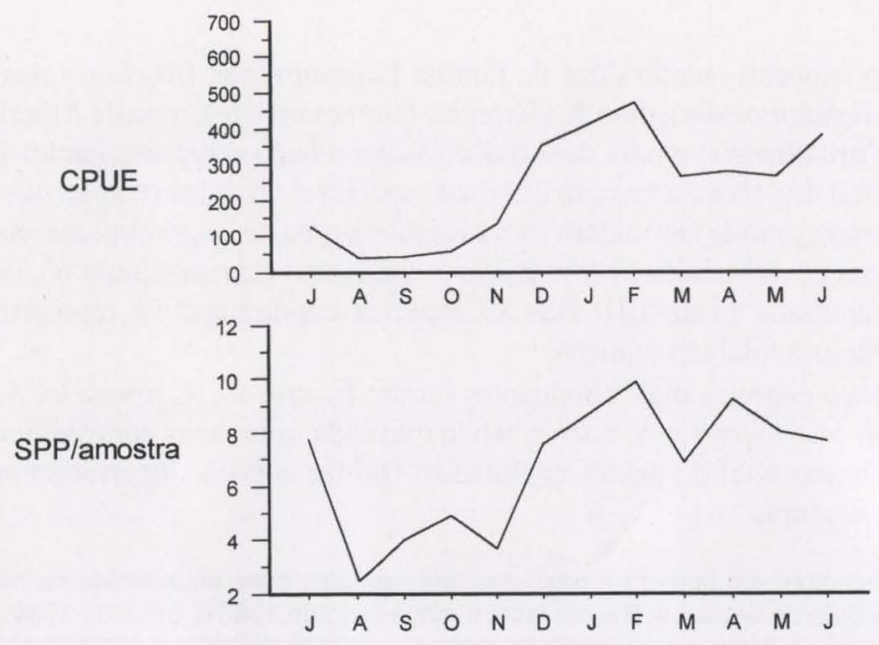

Fig. 1. Variação temporal da abundância relativa - CPUE (peixes/500 $\mathrm{m}^{2}$ ) e número de espécies, na margem continental da Baia de Sepetiba, Rio de Janeiro.

Gerres aprion e A. lepidentostole ocorreram principalmente durante o verão; $A$. januaria e $A$. brasiliensis, no outono; e $N$. barba, no inverno. É notório a distribuição de $A$. brasiliensis e $A$. januaria durante todo o ano, principalmente durante as estações mais quentes (verão e outono). Gerres aprion e A. januaria apresentaram diferenças significativas entre as estações do ano $(\mathrm{p}<0,05)(\mathrm{Tab}$. IV; Fig. 2a).

Não foram encontradas diferenças significativas na abundância de peixes entre as estações de coleta, embora o maior número de indivíduos tenha se verificado na zona externa da Baía de Sepetiba, com as estações de Muriqui (MU) e Itacuruçá (IT) contribuindo com um número total maior de indivíduos capturados (2.095 e 3.055 , respectivamente). O número total de indivíduos foi menor na estação da zona interna de Praia de Sepetiba (PS), contribuindo com um total de 1.364 peixes.

Uma clara tendência é apresentada em relação ao número de espécies, com um maior número de espécies tendo sida registrada na zona interna (estações PG e PS) e menor na zona externa, embora não se tenham encontrado diferenças significativas ao nivel de $95 \%$ de confiança $(p<0,05)$ entre as estações de coleta (Tab. IV; Fig. 3).

Considerando as espécies mais abundantes (contribuição acima de $4 \%$ do total de peixes capturados), não foram encontradas diferenças significativas entre os padrões de distribuição na margem continental da Baía de Sepetiba. No entanto, algumas tendências foram apresentadas, indicando separação espacial entre os grupos mais abundantes: A. januaria e $N$. barba dominaram nas estações da zona interna da Baía (PS e PG); $A$. brasiliensis para a estação de Coroa Grande (CG) e G. aprion e A. lepidentostole nas estações da zona externa (IT e MU). G. aprion e A. brasiliensis foram as espécies de distribuição mais ampla em todas as estações de coleta (Fig. 2b). 

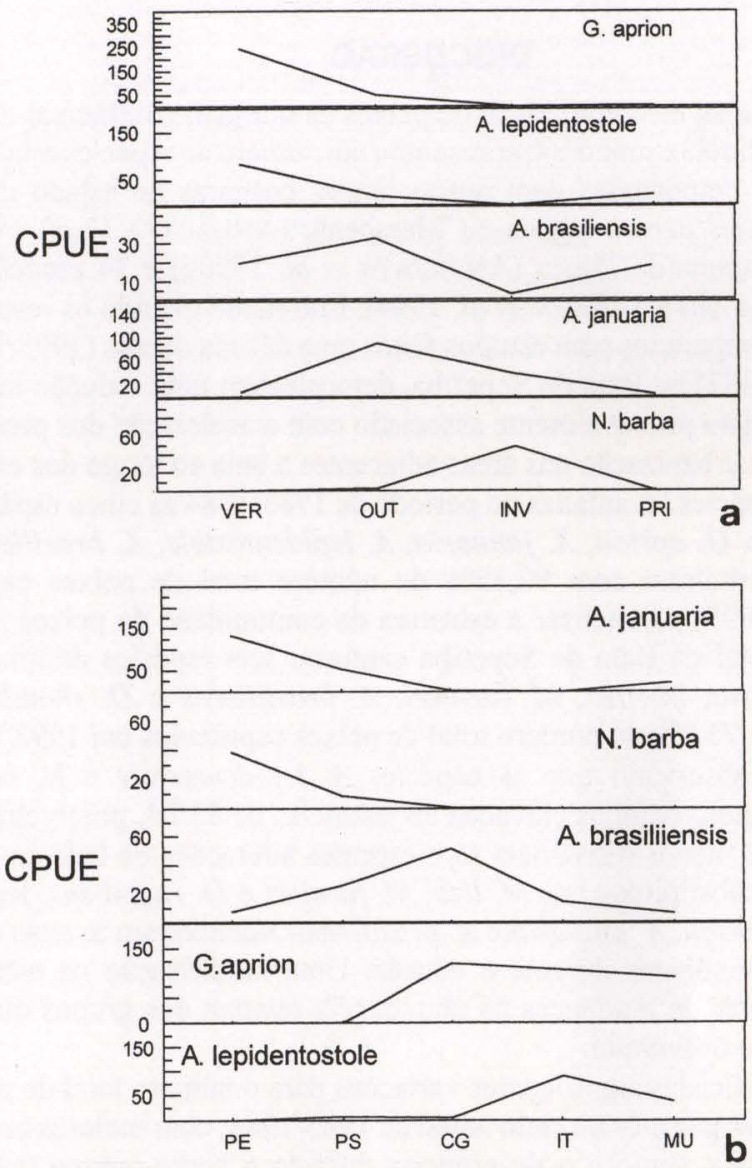

Fig. 2. Variação temporal (a) e espacial (b) das cinco espécies mais abundantes, por estação do ano na margem continental da Baía de Sepetiba, Rio de Janeiro.

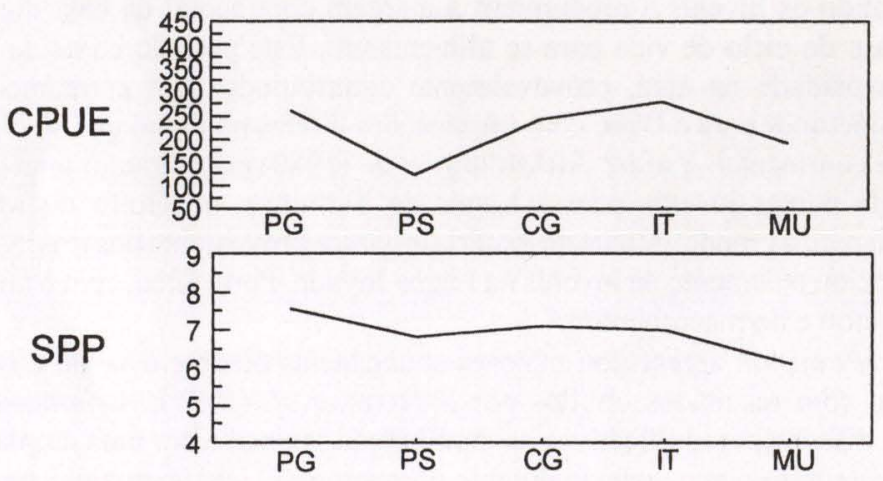

Fig. 3. Variação espacial da abundância relativa do númeor de indivíduos e número de espécies, na margem continental da Baía de Sepetiba, Rio de Janeiro. 


\section{DISCUSSÃO}

A composição da comunidade de peixes da margem continental da Baía de Sepetiba entre julho/ 83 e junho/84, apresentou um número de espécies relativamente alta (80) quando comparadas com outras lagoas costeiras do estado do Rio de Janeiro: 37 espécies para a laguna da Marapendi (ANDREATA et al. 1990a); 49 espécies para a laguna da Tijuca (ANDREATA et al. 1990b) e 34 espécies para o sistema lagunar de Maricá (BRUM et al. 1994). Entretanto quando os resultados de 1983/1984 são comparados com estudos feitos uma década depois (1993/1994) por ARAúJo et al. (1997) na Baía de Sepetiba, demostraram uma redução no número de espécies (55), isto provavelmente associado com a aceleração dos processos de industrialização e urbanização nas áreas adjacentes a baía ao longo dos anos.

Das 80 espécies levantadas no período de 1983/1984 as cinco espécies mais abundantes foram $G$. aprion, A. januaria, A. lepidentostole, A. brasiliensis e $N$. barba, que contribuíram com $92,15 \%$ do número total de peixes capturados. ARAÚJO et al. (1997) ao analisar a estrutura da comunidade de peixes jovens da margem continental da Baía de Sepetiba capturou seis espécies dominantes: $G$. aprion, A. januaria, M. liza, M. furnieri, A. brasiliensis e D. rhombeus, que contribuíram com 75,9\% do número total de peixes capturados em 1993/1994.

Pode ser observado que as espécies A. lepidentostole e $N$. barba em 1993/1994 não mantiveram as elevadas abundâncias de 83/84, possivelmente por serem espécies de menor resistência as crescentes alterações da baía ao longo da década, e foram substituídas por M. liza, M. furnieri e D. rhombeus. Ressalta-se também que $G$. aprion, A. januaria e $A$. brasiliensis mantiveram as suas contribuições relativas abundantes durante a década. Uma modificação na estrutura da comunidade, através de mudanças na abundancia relativa dos grupos mais abundantes foi portanto observada.

Foram verificadas significantes variações para o número total de peixes e $o$ número de espécies por mês no ciclo anual de 1983/1984, com maiores capturas de indivíduos durante o verão e o de espécies durante o verão-outono (sete a oito espécies por arrasto). Essas oscilações provavelmente estão associadas com a alta produtividade primária, uma característica encontrada em baías (ARAÚJO \& SANTOS 1999), levando os juvenis a procurarem a margem continental da baía durante a primeira fase do ciclo de vida para se alimentarem. Este período coincide com a maior pluviosidade na área, provavelmente contribuindo com carreamento de materiais alóctones para a Baía, com implicações diretas na fauna que se distribui na margem continental. YAÑEZ-ARANCIBIA et al. (1980) encontraram uma grande afluência de peixes juvenis para a Lagoa de Términos, no Golfo do México, coincidindo com a grande entrada de matéria orgânica proveniente dos rios; STONER (1986) associou o aumento de juvenis na Lagoa Joyuda, Porto Rico, com o aumento do zooplâncton e do macrobenton.

Gerres aprion apresentou maiores abundâncias durante o verão e outono, coincidindo com resultados obtidos por SANTOS et al. (1997); A. januaria não apresentou diferenças significativas na distribuição sazonal, com uma ampla ocorrência durante todo o ano, principalmente no outono; $A$. lepidentostole teve a sua maior abundância durante os meses do verão; $N$. barba nos meses de inverno e $A$. brasiliensis durante o verão e o outono. A ampla distribuição desta espécie tem sido 
reportado por SouZA \& ARAúJo (1990) e ARAúJo et al. (1997) para a Baía de Sepetiba, sendo citada também em diversos trabalhos que enfatizam a margem continental ao longo da costa sudeste-sul (PAIVA FILHO \& TOSCANO 1987; GIANNINI \& PAIVA FILHO 1995).

A distribuição espacial dos peixes no ciclo de 1983/1984 não apresentou diferenças significativas entre os locais de coleta, apesar de indicar um grande número de indivíduos para zona externa, principalmente na estação de Itacuruçá, um local de águas calmas proporcionada pela proteção da Ilha de Itacuruçá que atua como uma barreira, amenizando o impacto das correntes marinhas; e um maior número de espécies para zona interna da baía, principalmente a estação de Pedra de Guaratiba, um local com uma alta turbidez devido ao substrato lamoso e também maior carreamento de material orgânico proveniente das áreas urbanas adjacentes. BLABER \& WHITFIELD (1977) observaram que águas mais calmas podem ser um fator importante na distribuição de juvenis em estuários, enquanto BLABER \& BLABER (1980) discutem que a turbidez é um fator que proporciona uma diminuição da predação e um aumento de itens alimentares em águas rasas para os peixes juvenis. MCFARLAND (1963) salienta que há uma nítida relação entre a disponibilidade de alimento e a diversidade de espécies, sendo que um aumento na quantidade de alimento favorece uma maior especialização alimentar. No entanto, os resultados obtidos por ARAÚJO et al. (1997) indicam que a zona interna da baía contribui com maiores valores para o número total de espécies quanto para o número total de indivíduos. Essa diferenças observadas na distribuição espacial podem estar relacionadas a características que são peculiares a cada estação de coleta, sejam elas bióticas ou abióticas, gerando desta forma uma pequena diferença na composição e abundância das populações que habitam e/ou utilizam a área estudada.

Uma separação espacial das cinco espécies mais abundantes pode ser observada com $A$. lepidentostole mais distribuída nas estações da zona externa, $N$. barba na zona mais interna (PG), G. aprion ocorreu principalmente na estação de Coroa Grande (CG), e A. januaria e A. brasiliensis apresentaram uma distribuição ao longo de toda a margem continental, sendo maior a ocorrência destas na estação de Coroa Grande (CG). Essa separação também foi evidenciado por ARAÚJo et al. (1997) para $G$. aprion, A. januaria e A. brasiliensis, sendo esta última considerada como espécie residente da margem continental da Baía.

Estudos adicionais serão necessários para maior conhecimento da comunidade ictiofaunística da margem continental da Baía de Sepetiba. Sua análise contribui enormemente não só para o conhecimento das inúmeras espécies que ocorrem neste ambiente, mas também para a compreensão dos mecanismos de repartição que permitem que várias espécies coexistam neste ambiente, além de gerar subsídios para programas de utilização racional permitindo melhor aproveitamento dos recursos pesqueiros.

\section{REFERÊNCIAS BIBLIOGRÁFICAS}

ANDREATA, J.V.A. 1988. Revisão taxonômica do gênero Diapterus Ranzani 1840 (Pisces, Perciformes, Gerreidae). Acta Biol. Leopoldensia, São Leopoldo, 10 (1): 59-103.

- 1989. Estudo taxonômico da espécies de Gerres Quoy \& Gaimard, 1824 (Pisces, Perciformes, Gerreidae) que ocorrem em águas brasileiras. Acta Biol. Leopoldensia, São Leopoldo, 11 (1): 87-128. 
Andreata, J.V.A.; L.R.R. Barbieri; A.S.C. Sebília; M.H.C. Silva; M.A. Santos \& R.P. Santos. 1990a. Relação dos peixes da Laguna de Marapendi, Rio de Janeiro, Brasil. Atlântica, Rio Grande, 12 (1): 5-17.

ANDREATA, J.V.A.; A.M. SAAD; C.R.S. BIZERRIL \& F.A. BOCKMANN. 1990b. Alguns aspectos da ecologia das espécies de peixes da Laguna da Tijuca, período de março de 1987 a fevereiro de 1987. Acta Biol. Leopoldensia, São Leopoldo, 12 (2): 247-268.

AraúJo, F.G. \& A.C.A. SANTos. 1999. Distribuition and recruitment of mojarras (Perciformes, Gerreidae) in the continental margin of Sepetiba Bay, Brazil. Bull. Mar. Sc. 65 (2): 431-439.

Araúso, F.G.; A.G. Cruz-Filho; M.C.C. Azevedo; A.C.A. Santos \& L.A.M. Fernandes. 1997. Estrutura da comunidade de peixes jovens da margem continental da Baía de Sepetiba, RJ. Acta Biol. Leolpoldensia 19 (1): 61-83.

BENNETT, B.A. 1989. The fish community of moderately exposed beach on the south western Cape Coast of South Africa and an assesment of the habit as a nursery for juveniles fish. Estuarine Coastal Shelf Sci. 28 (9): 293-305.

BLABER, S.J.M. \& T.G. BLABER. 1980. Factores affeting the distribuition of juvenile estuarine and inshore fish. Jour. Fish. Biol. 17: 143-162.

BLABER, S.J.M. \& A.K. WHITFIELD. 1977. The feeding ecology of juvenile mugilidae in south east african estaries. Biol. Jour. Lin. Soc. 9: 227-284.

BRUM, M.J.I.; C.F.M.L. MuratoRI; P.R.D. LOPES \& P.R.F.G. VianNA. 1994. Ictiofauna do sistema lagunar de Maricá (RJ). Acta Biol. Leopoldensia, São Leopoldo, 16 (2): 45-55.

Carvalho, J.P.; L.R. Tomması \& M.D. Novelli. 1968. Lista de linguados no Brasil. Constr. Inst. Ocean. Univ. São Paulo, sér. Ocean. Biol., 14: 1-26.

DaY JR., J.W.; C.A.S. Hall; W.M. Kemp \& A. YÁNeZ-ARANCIBIA. 1989. Estuarine Ecology. New York, John Wiley and Sons Inc., 558p.

Figueiredo, J.L. \& N.A. Menezes. 1978. Manual de peixes marinhos do sudeste do Brasil. II. Teleostei (1). São Paulo, Museu de Zoologia, Universidade de São Paulo, 110p.

- 1980. Manual de peixes marinhos do sudeste do Brasil. III. Teleostei (2). São Paulo, Museu de Zoologia, Universidade de São Paulo, 90p.

FISCHER, W. 1978. FAO species indentification sheets for fishery purposes. Western Central Atlantic (Fishing Area 31), Vol. I-VII. Roma, FAO.

Giannini, R. \& A.M. PAiva FiLho. 1995. Análise comparativa da ictiofauna da zona de arrebentação de praias arenosas do estado de São Paulo, Brasil. Bol. Inst. Oceanogr., São Paulo, 43 (2): 141-152.

HoRN, M.H. 1980. Diel and season variation in abundance and diversity of shallow-water fish populations in Morro Bay, California. Fish. Bull. 78 (3): 759-770.

Livingston, R.J. 1982. Trophic organization of fishes in a costal seagrass system. Mar. Ecol. Prog. Ser. 7: 1-12.

MCFARLAND, W.N. 1963. Seasonal change in the number and biomass of fishes from the surf at Mustang Island, Texas. Publs Inst. Mar. Sci. Univ. Texas 9: 91-112.

Menezes, N.A. \& J.L. Figueiredo. 1980. Manual de peixes marinhos do sudeste do Brasil. IV. Teleostei (3). São Paulo, Museu de Zoologia, Universidade de São Paulo, 96p.

Menezes, N.A. \& J.L. Figueiredo. 1985. Manual de peixes marinhos do sudeste do Brasil. V. Teleostei (4). São Paulo, Museu de Zoologia, Universidade de São Paulo, 105p.

Oshiro, L.M.Y.O. \& F.G. ARaújo. 1987. Estudo dos peixes e crustáceos decápodes da Baía de Sepetiba, RJ. Simpósio Ecossistemas Costeiros do Sudeste-Sul do Brasil. Acad. Ciênc. São Paulo 3 (54): 283-297.

PaIVA FiLHo, A.M. \& A.P. TosCano. 1987. Estudo comparativo e variação da ictiofauna na zona entre marés do mar Casado-Guarujá e Mar Pequeno São Vicente, SP, Brasil. Relatório interno Inst. Oceanogr. Univ. São Paulo (17): 1-10.

Santos, A.C.A.; F.G. Araújo; A.G.C. Filho \& M.C.C. Azevedo. 1997. Distribuição e abundância relativa de Gerreidae (Osteichthyes, Perciformes) na Baía de Sepetiba, RJ. Arq. Biol. Tecnol., Rio de Janeiro, 4 (3): 672-686. 
SERGipenSE, S. \& I. SAzima. 1995. Variações sazonais de ocorrência e tamanho em duas espécies de Engraulididae(Osteichthyes) na Baía de Sepetiba, Rio de Janeiro. Rev. Brasil. Biol. 55 (3): 491-501. SOKAL, R.P. \& F.J. ROHLF. 1981. Biometry: the principales and pratice of statistic in biological research. San Francisco, W.H. Freman Co., $2^{\text {nd }}$ ed., 776p.

SouZA, D.C. \& F.G. ARAúJo. 1990. Distribuição e abundância do peixe-rei Xenomelaniris brasiliensis (Quoy \& Gaimard, 1824) na Baía de Sepetiba, RJ. IBAMA, Serviço de Defesa Ambiental DAS, Rio de Janeiro, 7: 1-12.

STONER, A.W. 1986. Communuty structure of demersal fish species of Laguna Joyuda, Puerto Rico. Estuaries 9 (2): 142-152.

Yañez-Arancibia, A.; F. Amezcua-Linares \& J.W. Day-JR. 1980. Fish community structure and function in Terminos Lagoon, a tropical estuary in the sourthen Gulf of Mexico, p. 465-482. In: V.S. Kennedy (Ed.). Estuarine Perspectives. New York, Academic Press, 495p.

Recebido em 29.IV.1999; aceito em 22.II.2000. 